\title{
Prospective comparative study of the influence of postoperative bile reflux on gastric mucosal histology and Campylobacter pylori infection
}

\author{
G J A OFFERHAUS, P $N$ M A RIEU, J B M J JANSEN, H J M JOOSTEN, \\ AND $C$ B $H$ W LAMERS
}

From the Departments of Gastroenterology-Hepatology and Pathology, University Hospital Leiden and the Department of Surgery, Canisius-Wilhelmina Hospital, Nijmegen, The Netherlands

SUMMARY Biopsies of 17 peptic ulcer patients, randomly treated by partial gastrectomy with either Billroth-II $(n=9)$ or Roux-en-Y $(n=8)$ anastomosis were studied before and six months after surgery to determine the role of bile reflux in the early postoperative histological alterations of the gastric mucosa. After BII-gastrectomy bile acid reflux (median 16.1 $\mu \mathrm{mol} / \mathrm{h}$ ) was significantly higher $(p<0 \cdot 0001)$ than after RY-gastrectomy $(0 \cdot 1 \mu \mathrm{mol} / \mathrm{h})$. Campylobacter pylori was present in the preoperative biopsies of all 17 patients. After RY-gastrectomy biopsies of all eight patients were positive for Campylobacter pylori, but was detected in only five of the nine patients with BIIgastrectomy. Preoperative scores of gastritis grading were similar in both groups and no significant differences were found postoperatively. Gastritis scores of the anastomotic mucosa in patients with BII-gastrectomy were significantly higher $(\mathbf{p}<\mathbf{0 . 0 2})$ than in the RY-gastrectomy group. Moreover, the reflux gastritis score in the four BII-gastrectomy patients cleared from Campylobacter pylori was significantly higher $(\mathbf{p}<0.02)$ than in the postgastrectomy patients harbouring Campylobacter pylori. The results suggest that reflux gastritis and Campylobacter pylori related gastritis are distinct microscopic entities and that bile reflux may play a role in the eradication of Campylobacter pylori after gastrectomy.

The gastric mucosa is repeatedly exposed to injurious agents of several kinds. Among others, postoperative reflux of bile is considered a factor of importance in the development of non-autoimmune chronic gastritis, the so called postoperative alkaline gastritis. '

Recent observations show that the gastric mucosa frequently harbours Campylobacter pylori, suggesting that non-autoimmune gastritis in some instances may also be an infectious disease. ${ }^{3}$ In fact, about $90 \%$ of peptic ulcer patients have active gastritis and infection of the gastric mucosa with Campylobacter pylori. Especially the discovery of Campylobacter pylori as a potential pathogenic microorganism for the human gastric mucosa led to renewed interest in

\footnotetext{
Address for correspondence: Prof Dr C B H W Lamers, Head, Dept Gastroenterology and Hepatology, Building 1, C4-P()15. University Hospital Leiden, PO Box 96(0), 230) RC Leiden. The Netherlands.

Accepted for publication 21 March 1989.
}

the different forms of gastritis and attempts to delineate their morphological features. ${ }^{+}$

Some recent reports point to specific histological characteristics of reflux gastritis, which differ from Campylobacter pylori related gastritis. ${ }^{5-7}$ Campylobacter pylori related gastritis appears to be closely associated with chronic active gastritis, indicated by the presence of mono and polymorphonuclear leucocytes. Delineation of the various morphological features, however, is almost always based upon retrospective examination of histopathological specimens with obvious drawbacks to define a histopathological entity. ${ }^{*}$ Moreover, the dynamics and evolution of inflammatory lesions in the postoperative gastric mucosa over time are well documented." Most study populations comprise nevertheless patients with postoperative intervals ranging from several months to several decades.

The purpose of our study therefore was prospect- 
ively investigate the early postoperative changes in the gastric mucosa of two basically comparable patient groups, randomly treated for peptic ulcer disease by a partial gastrectomy with either BillrothII or primary Roux-en-Y anastomosis, with preand postoperative assessment of the presence of Campylobacter pylori. A Roux-en-Y diversion was constructed to prevent postoperative reflux of bile, whereas a Billroth-II anastomosis is known to increase enterogastric reflux of bile. ${ }^{111}$

\section{Methods}

PATIENTS

Seventeen patients with peptic ulcer disease, not adequately responding to medical therapy, underwent elective gastrectomy. In all patients a two-thirds distal gastrectomy was performed and in random order a primary Roux-en-Y diversion or Billroth-II anastomosis was constructed. Nine patients underwent a partial gastrectomy with Billroth-II and eight patients a partial gastrectomy with Roux-en-Y anastomosis. Both groups were comparable with respect to mean age (52v47 $\mathrm{yr}$ ), sex (seven men and two women $v$ five men and three women) and indication for surgery (seven duodenal ulcer and two gastric ulcer $v$ six duodenal ulcer and two gastric ulcer). In the four gastric ulcer patients the ulcer was located at the angulus of the lesser curvature of the stomach. All patients were moderate to heavy smokers (10-20 cigarettes a day) at the time of operation. Six patients in the Billroth-II group and five in the Roux-en-Y group used moderate amounts $(<80 \mathrm{~g} /$ day $)$ of alcohol while none was treated by any medication at the time of the study. All the patients gave their informed consent to the study with preand postoperative endoscopy plus multiple gastric biopsies and quantitation of bile salts in the gastric aspirates. Endoscopies were performed one week preoperatively and six months postoperatively. The studies were approved by the local ethical committee on experimental investigations in man.

SAMPLING TECHNIQUE AND LABORATORY METHODS

After overnight fasting the patients underwent oesophagogastroduodenoscopy using the Olympus GIF-K fibre endoscope. At a separate occasion a nasogastric tube was installed intragastrically for measurement of reflux of bile salts for $60 \mathrm{~min}$. The gastric aspirates were divided into four 15 minute samples. After measurement of the volumes, the concentrations of bile salts were determined using the Enzabile test kit (Nygaard, Diagnostics Division Oslo, Norway). Fasting bile salt reflux was calculated for one hour, expressed as $\mu \mathrm{mol} / \mathrm{h}$.
Biopsy specimens were obtained using a forceps with spike and fenestrated cups. In each patient four biopsy specimens were taken preoperatively from the greater curvature of the antrum $5 \mathrm{~cm}$ from the pylorus and $4 \mathrm{~cm}$ from the corpus/fundus, two from the lesser curvature $5 \mathrm{~cm}$ distal from the cardia, and two from the greater curvature about half way cardiapylorus. Postoperatively, four stomal biopsies and four biopsies were obtained from the gastric remnant at similar sites as preoperatively. The biopsy specimens were orientated, immediately fixed in buffered formalin, and embedded in paraffin.

\section{HISTOLOGICAL ASSESSMENT OF BIOPSY}

SPECIMENS

Paraffin processed 4 micron sections were cut at different levels, stained by haematoxylin and eosin, and an additional section from each biopsy was stained by the Warthin Starry method for Campylobacter pylori. ${ }^{12}$

The sections were examined by one of us (GJAO), who was unaware of the operative procedure. The biopsies of antrum, lesser and greater curvature of corpus/fundus, and anastomosis were analysed separately. The activity and degree of gastritis at the various sites pre- and postoperatively were graded as nil ( 0$)$, mild (1), moderate (2), and severe (3) by (a) the degree of polymorphonuclear infiltration and (b) the degree of infiltration by chronic inflammatory cells and the severity of mucosal degeneration according to Whitehead et al. ${ }^{13}$ The most severe alteration in a section, formed the score for that specific variable at that site. The scores for the two variables were added, giving a maximum gastritis activity score of 6 . The specimens were also graded by the reflux gastritis score of Dixon et al, 5 from absent (0) to severe (3), for the following features: (a) foveolar hyperplasia, (b) oedema and smooth muscle fibres in the lamina propria, (c) vasodilatation and congestion of the lamina propria, and paucity of (d) acute and (e) chronic inflammatory cells. The highest score in a section for a certain variable was again considered representative for that specific variable at that biopsy site. A composite reflux gastritis score was calculated for each patient by adding the scores allotted for each of the individual variables (minimum score 0 , maximum score $15)$.

The sections stained by the Warthin Starry method were examined for the presence of Campylobacter pylori.

STATISTICAL ANALYSIS

Results were expressed as median and range. Statistical analysis was done by Wilcoxon's rank-sum test. Differences in the prevalence of Campylobacter 
pylori between the groups were tested for significance by the $\chi^{2}$ test.

\section{Results}

BILE REFLUX

Preoperative fasting bile salts in the gastric aspirates of the patients who ultimately underwent a BillrothII partial gastrectomy (median $0.35 \mu \mathrm{mol} / \mathrm{h}$, range $0.05-4.60 \mu \mathrm{mol} / \mathrm{h}$ ) were not significantly different from preoperative fasting bile salts in the patients who underwent a Roux-en-Y diversion (median 0.30 $\mu \mathrm{mol} / \mathrm{h}$, range $0 \cdot 00-4.00 \mu \mathrm{mol} / \mathrm{h}$ ). Postoperatively the fasting bile salt reflux was significantly higher $(\mathrm{p}<0.0001)$ after Billroth-II gastrectomy (median $16 \cdot 10 \mu \mathrm{mol} / \mathrm{h}$, range $0 \cdot 01-779 \cdot 27 \mu \mathrm{mol} / \mathrm{h}$ ) than after Roux-en-Y gastrectomy (median $0 \cdot 10 \mu \mathrm{mol} / \mathrm{h}$, range $0 \cdot 00-0 \cdot 31 \mu \mathrm{mol} / \mathrm{h}$ ).

\section{HISTOLOGICAL ASSESSMENT OF THE BIOPSY SPECIMENS}

\section{Gastritis activity score}

Table 1 shows the preoperative morphology of antrum and corpus graded according to Whitehead $e t$ $a l .{ }^{13}$ The microscopic picture was similar in patients who underwent BII-gastrectomy as compared with the patients who ultimately underwent Roux-en-Y diversion. Postoperatively, no statistically significant differences were found between the two groups, although the polymorphonuclear infiltration at the Billroth-II anastomosis was slightly less than in the Roux-en-Y patients. In addition, there were no significant differences between the results of pre and postoperative biopsies.

\section{Reflux gastritis score}

Table 2 shows the results of the reflux gastritis score

Table 1 Pre-and postoperative gastritis activity score (median and range) in patients with gastrectomy and Rouxen- $Y(n=8)$ or Billroth-II anastomosis $(n=9)$. The activity and degree of gastritis were graded as nil (O), mild (I), moderate (2), and severe (3) by (a) degree of polymorphonuclear infiltration and $(b)$ degree of infiltration by chronic inflammatory cells and the severity of mucosal degeneration. The gastritis score was calculated by adding the scores for the two parameters giving a maximum score of 6

\begin{tabular}{|c|c|c|c|c|}
\hline & \multicolumn{2}{|c|}{ Preoperative } & \multicolumn{2}{|c|}{ Postoperative } \\
\hline & Roux-en-Y & Billroth-II & Roux-en-Y & Billroth-II \\
\hline Antrum & $3 \cdot 5(2-6)$ & $4(2-5)$ & - & - \\
\hline \multicolumn{5}{|c|}{ Corpus/fundus } \\
\hline Greater curvaturs & $2 \cdot 5(1-4)$ & $2(0-4)$ & $3(1-5)$ & $2(1-4)$ \\
\hline Lesser curvature & $3(1-6)$ & $4(2-6)$ & $3(1-5)$ & $3(1-5)$ \\
\hline Anastomosis & - & - & $4(2-6)$ & $3(1-5)$ \\
\hline
\end{tabular}

Table 2 Histological reflux gastritis score (median and range) before and after gastrectomy, with either Roux-en-Y $(n=8)$ or Billroth-II $(n=9)$ anastomosis. The histological reflux parameters (a) foveolar hyperplasia, (b) oedema, (c) vasodilatation, (d) paucity of polymorphonuclear infiltration, and $(e)$ paucity of round cell infiltration were graded as nil (0), mild (1), moderate (2), and severe (3), giving a maximum cumulative reflux score of 15

\begin{tabular}{|c|c|c|c|c|}
\hline & \multicolumn{2}{|c|}{ Preoperative } & \multicolumn{2}{|c|}{ Postoperative } \\
\hline & Roux-en-Y & Billroth-II & $R o u x-e^{\prime} n-Y^{\prime}$ & Billroth-II \\
\hline $\begin{array}{l}\text { Antrum } \\
\text { Corpus/fundus }\end{array}$ & $4 \cdot 5(3-8)$ & $5(3-7)$ & - & - \\
\hline $\begin{array}{l}\text { Corpus/fundus } \\
\text { Greater curvature }\end{array}$ & $+(4-7)$ & $5(2-8)$ & $3 \cdot 5(2-6)$ & $5(4-10)$ \\
\hline Lesser curvature & $\begin{array}{l}+4(+-1) \\
3(2-5)\end{array}$ & $3(2-5)$ & $4 \cdot 5(2-7)$ & $5(3-9)$ \\
\hline Anastomosis & - & - & $5(3-8)$ & $9(5-1.3)^{*}$ \\
\hline
\end{tabular}

$\mathrm{p}<0 \cdot(12$ compared with Roux-en-Y.

as graded according to Dixon et al. ${ }^{5}$ The preoperative scores were not significantly different. Postoperatively, no significant differences were found for the greater and lesser curvature of the body of the stomach, although the score tended to be higher after Billroth-II gastrectomy. The characteristics of reflux gastritis, however, were more pronounced in the Billroth-II anastomosis than in the Roux-en-Y anastomosis and the composite reflux score in the Billroth-II group was significantly $(p<0.02)$ higher than in the Roux-en-Y group.

\section{Presence of Campylobacter pylori}

Campylobacter pylori was present in the preoperative mucosal biopsies of all 17 patients. After Rouxen-Y gastrectomy biopsies of all eight patients were still positive for Campylobacter pylori, whereas the microorganism was demonstrable in only five of the nine patients treated by a Billroth-II gastrectomy $\left(p=0 \cdot 06, \chi^{2}\right.$ test $)$. The four patients with Billroth-II gastrectomy whose mucosa was cleared from Campylobacter pylori had not been treated by antibiotics or colloidal bismuth in the period between the two endoscopies. Interestingly, both gastric ulcer patients who underwent gastrectomy with Billroth IIanastomosis were free of Campylobacter pylori in the postoperative biopsies. As shown in Figure 1, the reflux gastritis score was significantly $(\mathrm{p}<0 \cdot(02)$ higher in the four Campylobacter pylori negative patients as compared with the 13 Campylobacter pylori positive patients. The results of the gastric ulcer patients were not different from the patients operated upon for duodenal ulcer. In contrast, the gastritis activity score as shown in Figure 2, was significantly higher $(\mathrm{p}<0.02)$ in the 13 Campylobacter pylori positive patients than in the four 


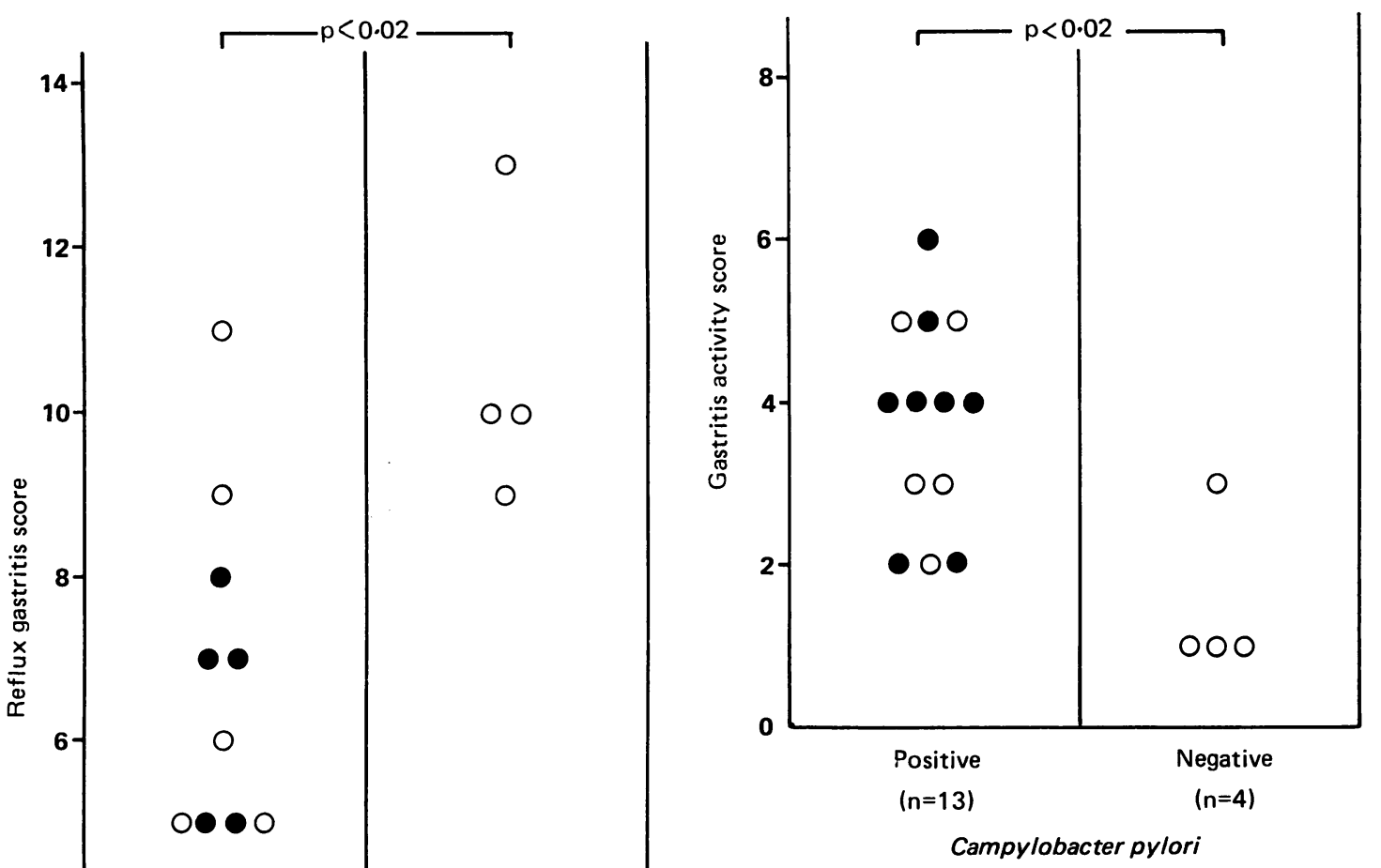

Fig. 2 Gastritis activity scores at the site of the anastomosis in 13 patients with persistent Campylobacter pylori infection and four patients whose gastric mucosa was cleared from Campylobacter pylori. Open circles denote Billroth-II anastomosis and closed circles Roux-en-Y diversion.

that bile reflux depends on the surgical procedure and the type of reconstruction performed after gastrectomy. The effect of diversion of bile on the histopathology of the gastric mucosa is more controversial. ${ }^{11+15}$ Surgical measures which proved to reduce bile reflux failed to show a difference for the histopathological picture of gastritis, "It making it questionable whether or not reflux of bile influences the architecture of the gastric mucosa at all. The poor correlation between histology and bile reflux could be partly explained by the fact that in all studies selected symptomatic patients with previous gastric surgery subsequently underwent a Roux-en-Y diversion. Furthermore, comparison of the degree of gastritis is almost without exception based on grades of chronic gastritis and inflammatory cell infiltration. ${ }^{11} 131+16$ Dixon et al recently postulated that reflux of bile is associated with a distinctive histopathological entity in which foveolar hyperplasia, vasodilatation, interstitial oedema and congestion, and paucity of inflammatory cells are the hallmarks. Foveolar hyperplasia as a characteristic of alkaline reflux gastritis has been emphasised by others ${ }^{171}$ and
Elimination of bile reflux by diversion of bile through a Roux-en-Y construction in patients with reflux gastritis is well documented." "I Our study confirms 
one of these reports also points to the relative absence of inflammatory cells. ${ }^{1 \mathrm{I}}$ It has to be stressed, however, that reflux gastritis is not a unique histological entity, as similar features can be seen in the neighbouring mucosa of chronic erosions, foveolar and hyperplastic polyps, Ménétrier's disease or gastritis cystica profunda. These conditions, however, were not present in the patients studied.

Redness and swelling of the gastric mucosa in reflux gastritis are well known macroscopic features for the clinician during endoscopy. "1 The microscopic analogues (hyperaemia, congestion, vasodilatation and oedema), may have been too little appreciated by histopathologists until now. ${ }^{5}$

Recognition of alkaline reflux gastritis as a separate microscopic picture has obvious clinical significance. When definition of this picture, however, is based on retrospective examination of histopathological material from outpatient departments or hospitals, it may be biased and not representative for alkaline reflux gastritis in general. ${ }^{\mathrm{x}}$ Furthermore, the evolution of inflammatory changes and the development of the histopathological features of postoperative reflux gastritis as a function of time should be taken into account. In most studies the intervals from the initial surgery show wide variations and no subdivision according to length of postoperative interval has been made, thus ignoring the dynamic principles of the behaviour of gastritis. ${ }^{8}$

The present study is, to our knowledge, the first randomised trial in which prospectively the influence of bile reflux on the human gastric mucosa was investigated. It confirms that, at least in the early postoperative stage, gastritis at the site of the anastomosis after Billroth-II resection seems to be characterised by specific features as recently

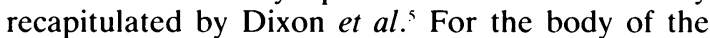
gastric remnant the higher reflux gastritis scores after Billroth-II resection failed to reach statistical significance when compared with biopsies from the respective sites preoperatively and after Roux-en-Y diversion. The finding that the reflux activity score at the anastomosis was increased, while the gastritis activity score was not affected by Billroth-II gastrectomy may seem to be contradictory, because infiltration of the gastric mucosa with inflammatory cells is a feature contributing oppositely to the scores of both classifications. The gastritis score according to Whitehead, however, depends heavily on infiltration with inflammatory cells, whereas paucity of these cells in the gastric mucosa contributes only for $40 \%$ to the reflux activity score. Thus, the increase in reflux score was mainly caused by other factors, such as foveolar hyperplasia, oedema and vasodilatation. Furthermore, there was in fact a weak tendency to a decreased density of inflammatory cells postopera- tively, leading to a slightly lower gastritis score after Billroth-II gastrectomy, while there was a minimal increase of this score after Roux-en-Y gastrectomy (Table 1). These changes of inflammatory cells may be in part secondary to the mucosal swelling after Billroth-II gastrectomy.

The postoperative findings in our Billroth-II study group with respect to Campylobacter pylori status, indicate a potential role of bile reflux in the eradication of these microorganisms. Other studies, in which the prevalence of Campylobacter pylori infection after partial gastrectomy was also lower than expected, support this observation. ${ }^{7710}$ As the patients had not been treated by antibiotics or colloidal bismuth, it is likely that alkaline reflux into the gastric remnant played a causative role in the clearance of the microorganism.

We conclude from this prospective comparative study that the type of anastomosis after gastric resection is an important determinant of mucosal histology evaluated by a reflux gastritis score and persistence or clearance of Campylobacter pylori infection.

The authors are indebted to $\mathrm{J} \mathrm{B}$ de Boer for technical support, to Ir I Biemond for preparing the drawings and to Lya Liefting and Loes Niepoth for secretarial assistance.

\section{References}

1 Ritchic WP. Alkaline reflux gastritis: an objective assessment of its diagnosis and treatment. Ann Surg 1980; 192: 288-98.

2 Ritchic WP. Alkaline reflux gastritis: a critical reappraisal. Gut 1984; 25: 975-87.

3 Blaser MJ. Gastric Campylobacter-like organisms, gastritis and peptic ulcer discase. Gastroenterology 1987; 93: 371-83.

4 Wyatt JI. Dixon MF. Chronic gastritis. A pathogenetic approach. J Pathol 1988; 154: 123-4.

5 Dixon MF, O’Connor HJ, Axon ATR, King RFGJ, Johnston D. Reflux gastritis - a distinct histopathological entity? J Clin Pathol 1986; 39: 524-30.

6 O'Connor HJ, Wyatt JI, Ward DC, et al. Effect of duodenal ulcer surgery and enterogastric reflux on Campylobacter pyloridis. Lancet 1986; ii: 1178-81.

7 O’Connor HJ, Newbold KM, Drumm J, Donovan IA. Thompson H. Alexander-Williams J. Effect of bile diversion on Campylobacter pylori [Abstract]. Gut 1988; 29: A713.

8 Siurala M. Varis K, Kekki M. New aspects on epidemiology, genetics and dynamics of chronic gastritis. Front Gastrointest Res 1980; 6: 148-66.

9 Kekki M, Saukkonen M. Sipponen P, Varis K. Siurala M. Dynamics of chronic gastritis in the remnant after partial gastrectomy for duodenal ulcer. Scand $J$ Gastroenterol 1980; 15: 509-12. 
10) Kennedy T, Green R. Roux diversion for bile reflux following gastric surgery. Br J Surg 1978; 65: 323-5.

11 Malagelada JR, Philips SF, Shorter RG, et al. Postoperative reflux gastritis: pathophysiology and long term outcome after Roux-en-Y diversion. Ann Intern Med 1985: 103: 178-83.

12 Warthin AS, Starry AC. A more rapid and improved method of demonstrating spirochetes in tissues. $A m J$ Syphillis 1920; 4: 97-103.

13 Whitehead R, Truelove SC, Gear MWL. The histological diagnosis of chronic gastritis in fibreoptic gastroscope biopsy specimens. J Clin Pathol 1972: 25: $1-11$.

14 Hoare AM, McLeish A, Thompson H, AlexanderWilliams J. Selection of patients for bile diversion surgery: use of bile acid measurement in fasting gastric aspirates. Gut 1978; 19: 163-5.

15 Watt PCH, Sloan JM, Spencer A, Kennedy TL.
Histology of the postoperative stomach before and after diversion of bile. Br Med J 1983; 287: 1410-2.

16 Meshkipour H. Marks JW. Schocnficld LJ. Bonnoris GG, Carter S. Reflux gastritis syndrome: mechanism of symptoms. Gastroenterology 1980; 79: 1283-7.

17 Bechi P. Amorosi A. Mazzanti R. Romagnoli P. Tonelli L. Gastric histology and fasting bile reflux after partial gastrectomy. Gastroenterology 1987: 93: 335-43.

18 Weinstein WM. Buch KL. Elashoff J, et al. The histology of the stomach in symptomatic patients after gastric surgery: a model to assess selective patterns of gastric mucosal injury. Scand J Gastroenterol 1985; 20 (suppl 109): 77-89.

19 Gustavsson S. Phillips S. Malagelada JR. Rosenblatt JE. Assessment of Campylobacter-like organisms in the postoperative stomach, iatrogenic gastritis and chronic gastroduodenal diseases: preliminary observations. Mayo Clin Proc 1987: 62: 26.5-8. 